\title{
2-D FIR Eigenfilters: A Least-Squares Approach
}

\author{
SOO-CHANG PEI, MEMBER, IEEE, AND JONG-JY SHYU, STUDENT MEMBER, IEEE
}

\begin{abstract}
The 1-D eigenfilter approach is extended for designing 2-D FIR filters. By minimizing a quadratic measure of the error in the 2-D frequency band, an eigenvector of an appropriate matrix is computed to get the filter coefficients. This method is not only simple but is also optimal in the least-squares sense. Several numerical design examples of 2-D arbitrary shape filters are used to illustrate the effectiveness of this approach.
\end{abstract}

\section{INTRODUCTION}

$\mathbf{R}$ ECENTLY, Vaidyanathan and Nguyen introduced a new method for the design of 1-D linear phase FIR digital filters, referred to as the eigenfilter method [1], which involves minimizing a quadratic measure of the error in the passband and stopband. The method is based on the computation of an eigenvector of a quadratic measure of the error in the passband and stopband. The method is based on the computation of an eigenvector of a real, symmetric, and positive-definite matrix; the resultant eigenvector corresponding to the smallest eigenvalue is the desired filter coefficient. The advantage of the eigenfilter approach over the McClellan-Parks algorithm [2] is that it is general enough to incorporate both time- and frequency-domain constraints.

In this paper, the 1-D eigenfilter approach is extended for designing 2-D quadrantal symmetric FIR filters. A similar idea is independently developed by Nashashibi and Charalambous [3]. The method is proposed by minimizing a quadratic measure of the passband and/or stopband error in the 2-D frequency domain. The total error function can be formulated as

$$
\begin{aligned}
E= & \alpha \int_{p} \int\left[D\left(w_{1}, w_{2}\right)-\hat{H}\left(w_{1}, w_{2}\right)\right]^{2} d w_{1} d w_{2} \\
& +\beta \iint\left[\hat{H}\left(w_{1}, w_{2}\right)\right]^{2} d w_{1} w_{2} \\
= & \alpha E_{p}+\beta E_{s}
\end{aligned}
$$

where

$D\left(w_{1}, w_{2}\right)$ desired amplitude response,

$\hat{H}\left(w_{1}, w_{2}\right)$ actual amplitude response of the 2-D filter $H\left(Z_{1}, Z_{2}\right)$,
$E_{p} \quad$ passband error,

$E_{s} \quad$ stopband error,

$p \quad$ passband region,

$s \quad$ stopband region,

$\alpha, \beta \quad$ weighting constants which control the relative accuracies of approximation in the passband and stopband, respectively. In general, the larger the parameter, the better the performance of its corresponding band. $\alpha$ and $\beta$ can also be a function $w_{1}$ and/or $w_{2}$.

Equation (1) can be reformulated properly such that it has the following form:

$$
E=A^{t} Q A
$$

where $t$ is the vector transpose operation, $Q$ is a real, symmetric and positive-definite matrix, and $A$ is a real vector related to the 2-D filter impulse response $h\left(n_{1}, n_{2}\right)$ in some manner. By Rayleigh principle [4], the eigenvector $A$ associated with the smallest eigenvalue of matrix $Q$ minimizes the total error $E$.

This approach is optimal in the least square sense, and is flexible for designing 2-D FIR filters with additional timeand/or frequency-domain constraints. In Section II, we formulate the quadratic function of error and present some design examples such as 2-D circular low-pass filters, fan filters, etc. Then time- and frequency-domain constraints are incorporated into the error function in Section III to demonstrate the flexibility and capability of this approach. Section IV gives a new definition of 2-D half-band filter extended from 1-D half-band filter and includes a design example using the eigenfilter approach. In Section $\mathrm{V}$, we make a detailed comparison of this method with the wellknown McClellan's transformation method [5] in terms of passband ripple, stopband attenuation, filter contour and design time, and discuss the choice of the reference frequency point; We also extend the eigenfilter method to design a 3-D circular symmetric low-pass filter very easily. Finally, Section V gives a summary.

\section{2-D QuAdRANTAL Symmetric Eigenfilter Design}

A 2-D FIR digital filter with transfer function $H\left(Z_{1}, Z_{2}\right)$ and the impulse response $h\left(n_{1}, n_{2}\right)$ has a frequency re- 
sponse

$$
H\left(e^{j w_{1}}, e^{j w_{2}}\right)=\sum_{n_{1}=0}^{N_{1}-1} \sum_{n_{2}=0}^{N_{2}-1} h\left(n_{1}, n_{2}\right) e^{-j n_{1} w_{1}} e^{-j n_{2} w_{2}}
$$

For a 2-D quadrantal symmetric filter with $N_{1}$ and $N_{2}$ odd, its impulse response $h\left(n_{1}, n_{2}\right)$ has the following relationship:

$$
\begin{aligned}
h\left(\frac{N_{1}-1}{2}-\right. & \left.k_{1}, \frac{N_{2}-1}{2}-k_{2}\right) \\
& =h\left(\frac{N_{1}-1}{2}-k_{1}, \frac{N_{2}-1}{2}+k_{2}\right) \\
& =h\left(\frac{N_{1}-1}{2}+k_{1}, \frac{N_{2}-1}{2}-k_{2}\right) \\
= & h\left(\frac{N_{1}-1}{2}+k_{1}, \frac{N_{2}-1}{2}+k_{2}\right), \\
& 1 \leqslant k_{1} \leqslant \frac{N_{1}-1}{2}, \quad 1 \leqslant k_{2} \leqslant \frac{N_{2}-1}{2}
\end{aligned}
$$

then (3) can be written in the form [6]:

$$
\begin{aligned}
H\left(e^{j w_{1}}, e^{j w_{2}}\right)= & e^{-j \frac{N_{1}-1}{2} w_{1}} e^{-j \frac{N_{2}-1}{2} w_{2}} \\
& \frac{N_{1}-1}{2} \frac{N_{2}-1}{2} \\
& \cdot \sum_{n_{1}=0}^{2} \sum_{n_{2}=0} a\left(n_{1}, n_{2}\right) \cdot \cos n_{1} w_{1} \cos n_{2} w_{2} \\
= & e^{-j \frac{N_{1}-1}{2} w_{1}} e^{-j \frac{N_{2}-1}{2} w_{2}} \cdot \hat{H}\left(w_{1}, w_{2}\right)
\end{aligned}
$$

where $\hat{H}\left(w_{1}, w_{2}\right)$ is the amplitude response of $H\left(e^{j w_{1}}, e^{j w_{2}}\right)$, and $a\left(n_{1}, n_{2}\right)$ is related to the filter impulse response $h\left(n_{1}, n_{2}\right)$ by
Hence we can write the amplitude response $\hat{H}\left(w_{1}, w_{2}\right)$ as

$$
\hat{H}\left(w_{1}, w_{2}\right)=A^{t} \cdot C\left(w_{1}, w_{2}\right)
$$

Notice that $A$ and $C\left(w_{1}, w_{2}\right)$ are both column vectors; Then from (1) and (8), the stopband error can be defined as

$$
\begin{aligned}
E_{s} & =\int_{s} \int\left[A^{t} C\left(w_{1}, w_{2}\right)\right] \cdot\left[A^{t} \cdot C\left(w_{1}, w_{2}\right)\right]^{t} d w_{1} w_{2} \\
& =A^{t}\left[\iint_{s} C\left(w_{1}, w_{2}\right) \cdot C^{t}\left(w_{1}, w_{2}\right) d w_{1} d w_{2}\right] A \\
& =A^{t} Q_{s} A
\end{aligned}
$$

and the passband error is

$$
\begin{aligned}
E_{p}= & A^{t}\left\{\int_{p} \int\left[\frac{D\left(w_{1}, w_{2}\right)}{D\left(w_{10}, w_{20}\right)} C\left(w_{10}, w_{20}\right)-C\left(w_{1}, w_{2}\right)\right]\right. \\
& \left.\cdot\left[\frac{D\left(w_{1}, w_{2}\right)}{D\left(w_{10}, w_{20}\right)} \cdot C\left(w_{10}, w_{20}\right)-C\left(w_{1}, w_{2}\right)\right]^{t} d w_{1} d w_{2}\right\} A \\
= & A^{t} Q_{p} A
\end{aligned}
$$

where $\left(w_{10}, w_{20}\right)$ is the reference frequency point in the passband we choose to approach the passband to this desired reference frequency response.

This enables us to write the total error $E$ as a quadratic in $A$, this will lead to the eigenformulation $E=A^{t} Q A$, and the matrix $Q$ is

$$
Q=\alpha Q_{p}+\beta Q_{s}
$$

where $\alpha$ and $\beta$ are weighting contants. For $N$ odd, the size of matrix $Q$ is $((N+1) / 2)^{2} \times((N+1) / 2)^{2}$, and the ele-

$$
\begin{aligned}
a(0,0) & =h\left(\frac{N_{1}-1}{2}, \frac{N_{2}-1}{2}\right) ; a\left(0, n_{2}\right)=2 h\left(\frac{N_{1}-1}{2}, \frac{N_{2}-1}{2}-n_{2}\right), & & n_{2}=1, \cdots, \frac{N_{2}-1}{2} \\
a\left(n_{1}, 0\right) & =2 h\left(\frac{N_{1}-1}{2}-n_{1}, \frac{N_{2}-1}{2}\right), & & n_{1}=1, \cdots, \frac{N_{1}-1}{2} \\
a\left(n_{1}, n_{2}\right) & =4 h\left(\frac{N_{1}-1}{2}-n_{1}, \frac{N_{2}-1}{2}-n_{2}\right), & & n_{1}=1, \cdots, \frac{N_{1}-1}{2} ; n_{2}=1, \cdots, \frac{N_{2}-1}{2} .
\end{aligned}
$$

For simplicity, let $N_{1}=N_{2}=N$ and define a column vector as

$A=\left[a(0,0), a(0,1), \cdots, a\left(0, \frac{N-1}{2}\right): a(1,0), \cdots, a\left(1, \frac{N-1}{2}\right): \cdots: a\left(\frac{N-1}{2}, 0\right), a\left(\frac{N-1}{2}, 1\right), \cdots, a\left(\frac{N-1}{2}, \frac{N-1}{2}\right)\right]^{\iota}$

and

$$
\begin{array}{r}
C\left(w_{1}, w_{2}\right)=\left[1, \cos w_{2}, \cdots, \cos \frac{N-1}{2} w_{2} \cos w_{1}, \cos w_{1} \cdot \cos w_{2}, \cdots, \cos w_{1} \cdot \cos \frac{N-1}{2} w_{2} \cos \frac{N-1}{2} w_{1},\right. \\
\left.\cos \frac{N-1}{2} w_{1} \cdot \cos w_{2}, \cdots, \cos \frac{N-1}{2} w_{1} \cdot \cos \frac{N-1}{2} w_{2}\right]^{t} .
\end{array}
$$


ments of $Q$ are given by

$$
\begin{aligned}
q(n, m)= & \alpha \int_{p} \int\left[\frac{D\left(w_{1}, w_{2}\right)}{D\left(w_{10}, w_{20}\right)} \cos n_{1} w_{10} \cos n_{2} w_{20}-\cos n_{1} w_{1} \cdot \cos n_{2} w_{2}\right] \\
& \cdot\left[\frac{D\left(w_{1}, w_{2}\right)}{D\left(w_{10}, w_{20}\right)} \cos m_{1} w_{10} \cos m_{2} w_{20}-\cos m_{1} w_{1} \cdot \cos m_{2} w_{2}\right]^{t} d w_{1} d w_{2} \\
& +\beta \iint\left[\cos n_{1} w_{1} \cos n_{2} w_{2} \cos m_{1} w_{1} \cos m_{2} w_{2}\right] d w_{1} d w_{2}
\end{aligned}
$$

where $1 \leqslant n, m \leqslant(N B)^{2}, N B=(N+1) / 2$.

$$
n_{1}=\bmod (n-1, N B) \text { and } n_{2}=\text { integer part of } \frac{n-1}{N B}
$$

and

$$
m_{1}=\bmod (m-1, N B) \text { and } m_{2}=\text { integer part of } \frac{m-1}{N B}
$$

Once the matrix $Q$ is obtained according to the design requirement, we can compute the eigenvector $A$ which corresponds to the smallest eigenvalue, it is easy to get the filter impulse response $h\left(n_{1}, n_{2}\right)$ from the eigenvector $A$ by (6).

A similar procedure can be applied for the 2-D case for $N$ even. The amplitude response in this case is

$$
\begin{array}{r}
\hat{H}\left(w_{1}, w_{2}\right)=\sum_{n_{1}=1}^{N / 2} \sum_{n_{2}=1}^{N / 2} a\left(n_{1}, n_{2}\right) \cos \left(n_{1}-1 / 2\right) w_{1} \\
\cdot \cos \left(n_{2}-1 / 2\right) w_{2}
\end{array}
$$

where

$$
\begin{aligned}
a\left(n_{1}, n_{2}\right)=4 h\left((N / 2)-n_{1},(N / 2)-n_{2}\right), & \\
& n_{1}, n_{2}=1,2, \cdots, N / 2 .
\end{aligned}
$$

\section{Example 2.1. Design of 2-D Rectangular Low-Pass Filter}

In this example, we want to design a $2-\mathrm{D}$ rectangular low-pass filter whose specification is shown in Fig. 1(a), where $w_{p 1}, w_{s 1}$ and $w_{p 2}, w_{s 2}$ represent the passband and stopband cutoff frequencies in the $w_{1}$ and $w_{2}$ axes, respectively. For this design if we take the origin of the passband as the reference frequency point, i.e., $\left(w_{10}, w_{20}\right)=(0 ., 0$.$) ,$ then $(10)$ is easy to express it in closed form; Fig. 1(b) shows a $27 \times 272-\mathrm{D}$ square low-pass filter with $w_{p 1}=w_{p 2}$ $=0.4 \pi, w_{s 1}=w_{s 2}=0.6 \pi$. The filter deviation and design time, including those of all other examples are summarized and tabulated in Table $\mathrm{I}$ and discussed in Section V.

\section{Example 2.2: Design of 2-D Circular/Elliptical} Low-Pass Filter

Fig. 2(a) shows the specification for designing a 2-D circular/elliptical low-pass filter in which the passband edge is described by the following relation:

$$
\left(w_{1} / w_{p 1}\right)^{2}+\left(w_{2} / w_{p 2}\right)^{2}=1
$$

and the stopband edge is related as

$$
\left(w_{1} / w_{s 1}\right)^{2}+\left(w_{2} / w_{s 2}\right)^{2}=1 .
$$

If $w_{p 1}=w_{p 2}$ and $w_{s 1}=w_{s 2}$, then it becomes a circular low-pass filter, for a circular filter design it is difficult to express (10) in a closed form, hence the numerical integration method is used to approximate the integral in (10). Fig. 2(b) presents the magnitude response of a 2-D circular low-pass filter with $N_{1}=N_{2}=25, w_{p 1}=w_{p 2}=0.5 \pi, w_{s 1}=$ $w_{s 2}=0.7 \pi$, and $\alpha=\beta=1$. We also use $(0 ., 0$.) as the reference frequency point.

\section{Example 2.3: Design of 2-D Fan Type Filter.}

Fig. 3(a) presents the specification of a 2-D fan filter in which $w_{s}$ is the stopband cutoff frequency. Setting $N_{1}=N_{2}$ $=23, w_{s}=0.16 \pi, \alpha=\beta=1$, and $\left(w_{10}, w_{20}\right)=(0, \pi / 2)$, we get the fan filter amplitude response shown in Fig. 3(b).

\section{Example 2.4: Design of 2-D Filters with Minimum Energy Stopbands}

Designing such filters, we only consider the stopband attenuation by setting $\alpha=0, \beta=1$ [1]. The response shown in Fig. 4 is that of a $15 \times 152-\mathrm{D}$ filter with $-52.21-\mathrm{dB}$ minimum energy stopband whose don't care passband is a circular region with radius $0.3 \pi$.

\section{2-D Eigenfilters with Simultaneous Time- AND FREQUENCY-DOMAIN CONSTRAINTS}

Error functions that are more general than (1) can be formulated in order to take into account time- and/or frequency-domain constraints. In this section, we will demonstrate the capability of the present approach by means of three examples.

\section{Example 3.1: Design of 2-D Elliptical Low-Pass Filters which can Reject Unwanted Known Signal}

For simplicity, we like to design the filter which can reduce occasional unwanted input signal with the waveform:

$$
s(i, j), \quad 0 \leqslant i, j \leqslant L-1
$$

and its corresponding filter's output $r\left(n_{1}, n_{2}\right)$ is

$$
\begin{array}{r}
r\left(n_{1}, n_{2}\right)=\sum_{k_{1}=0}^{N-1} \sum_{k_{2}=0}^{N-1} h\left(k_{1}, k_{2}\right) s\left(n_{1}-k_{1}, n_{2}-k_{2}\right), \\
0 \leqslant n_{1}, n_{2} \leqslant N+L-2 .
\end{array}
$$


Let

$$
\begin{gathered}
R=[r(0,0), r(0,1), \cdots, r(0, N+L-2) \vdots r(1,0), r(1,1), \cdots, r(1, N+L-2) ! \cdots \\
\cdots(N+L-2,0), \cdots, \\
r(N+L-2, N+L-2)]^{t} \\
H=[h(0,0), h(0,1), \cdots, h(0, N-1) \vdots h(1,0), h(1,1), \cdots, h(1, N-1) ; \cdots \\
h(N-1,0), \cdots, \\
h(N-1, N-1)]^{t}
\end{gathered}
$$

and

$$
S=\left[\begin{array}{lllllll}
s_{0} & 0_{1} & 0_{1} & \cdots & 0_{1} & 0_{1} & 0_{1} \\
s_{1} & s_{0} & 0_{1} & \cdots & 0_{1} & 0_{1} & 0_{1} \\
s_{2} & s_{1} & s_{0} & \cdots & 0_{1} & 0_{1} & 0_{1} \\
\vdots & \vdots & \vdots & \ddots & \vdots & \vdots & \vdots \\
s_{L-1} & s_{L-2} & s_{L-3} & \cdots & s_{0} & 0_{1} & 0_{1} \\
0_{1} & s_{L-1} & s_{L-2} & \cdots & s_{1} & s_{0} & 0_{1} \\
0_{1} & 0_{1} & s_{L-1} & \cdots & s_{2} & s_{1} & s_{0} \\
\vdots & \vdots & \vdots & \ddots & \vdots & \vdots & \vdots \\
0_{1} & 0_{1} & 0_{1} & \cdots & s_{L-1} & s_{L-2} & s_{L-3} \\
0_{1} & 0_{1} & 0_{1} & \cdots & 0_{1} & s_{L-1} & s_{L-2} \\
0_{1} & 0_{1} & 0_{1} & \cdots & 0_{1} & 0_{1} & s_{L-1}
\end{array}\right]
$$

where $0_{1}$ denotes a zero matrix with dimension $(N+L-1) \times N$ and

$$
S_{i}=\left[\begin{array}{lllllll}
s(i, 0) & 0 & 0 & \cdots & 0 & 0 & 0 \\
s(i, 1) & s(i, 0) & 0 & \cdots & 0 & 0 & 0 \\
s(i, 2) & s(i, 1) & s(i, 0) & \cdots & 0 & 0 & 0 \\
\vdots & \vdots & \vdots & \ddots & \vdots & \vdots & \vdots \\
s(i, L-1) & s(i, L-2) & s(i, L-3) & \cdots & s(i, 0) & 0 & 0 \\
0 & s(i, L-1) & s(, L-2) & \cdots & s(i, 1) & s(i, 0) & 0 \\
0 & 0 & s(i, L-1) & \cdots & s(i, 2) & s(i, 1) & s(i, 0) \\
\vdots & \vdots & \vdots & \ddots & \vdots & \vdots & \vdots \\
0 & 0 & 0 & \cdots & s(i, L-1) & s(i, L-2) & s(i, L-3) \\
0 & 0 & 0 & \cdots & 0 & s(i, L-1) & s(i, L-2) \\
0 & 0 & 0 & \cdots & 0 & 0 & s(i, L-1)
\end{array}\right]
$$

Equation 14 can be expressed in a matrix form:

$$
R=S \cdot H
$$

in which the dimension of $S$ is $(N+L-1)^{2} \times N^{2}$ and that of $S_{i}$ is $(N+L-1) \times N$. Now define a normalized measure of output noise by

$$
E_{N}=\frac{H^{t} S^{t} S H}{\hat{s}^{t} \cdot \hat{s}}
$$

where

$$
\hat{s}=[s(0,0), s(0,1), \cdots, s(0, L-1) \vdots s(1,0), \cdots, s(1, L-1): \cdots, s(L-1,0), \cdots, s(L-1, L-1)]^{t} .
$$

Moreover, $H$ is related to $A$ in (7a) for $N$ odd by

$$
H=D A
$$




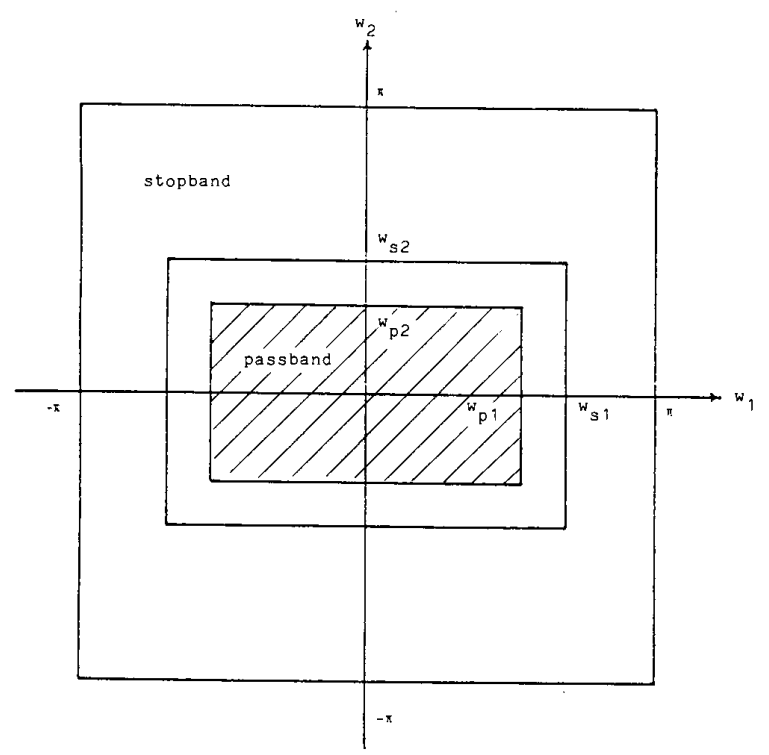

(a)

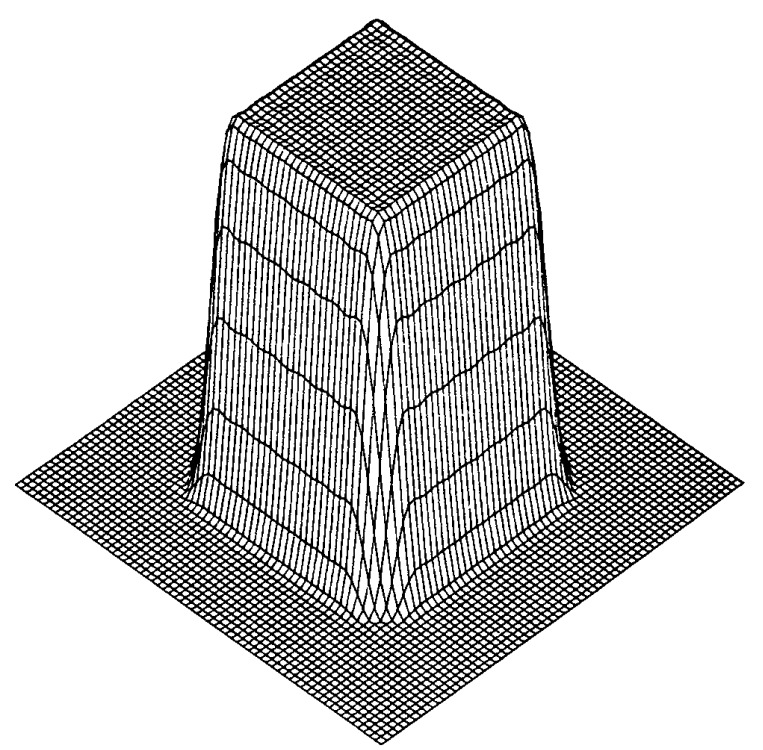

(b)

Fig. 1. (a) Specification of 2-D rectangular low-pass filter in Example 2.1. (b) A $27 \times 272$-D square low-pass filter with $w_{p 1}=w_{p 2}=0.4 \pi$, $w_{s 1}=w_{s 2}=0.6 \pi$, and $\alpha=\beta=1$ in Example 2.1.

where

$$
D=\left[\begin{array}{lllll}
0_{2} & 0_{2} & 0_{2} & \ldots & D_{1} \\
\vdots & \vdots & \vdots & \ddots & \vdots \\
0_{2} & 0_{2} & D_{1} & \cdots & 0_{2} \\
0_{2} & D_{1} & 0_{2} & \ldots & 0_{2} \\
2 D_{1} & 0_{2} & 0_{2} & \ldots & 0_{2} \\
0_{2} & D_{1} & 0_{2} & \ldots & 0_{2} \\
0_{2} & 0_{2} & D_{1} & \cdots & 0_{2} \\
\vdots & \vdots & \vdots & \ddots & \vdots \\
0_{2} & 0_{2} & 0_{2} & \cdots & D_{1}
\end{array}\right]
$$

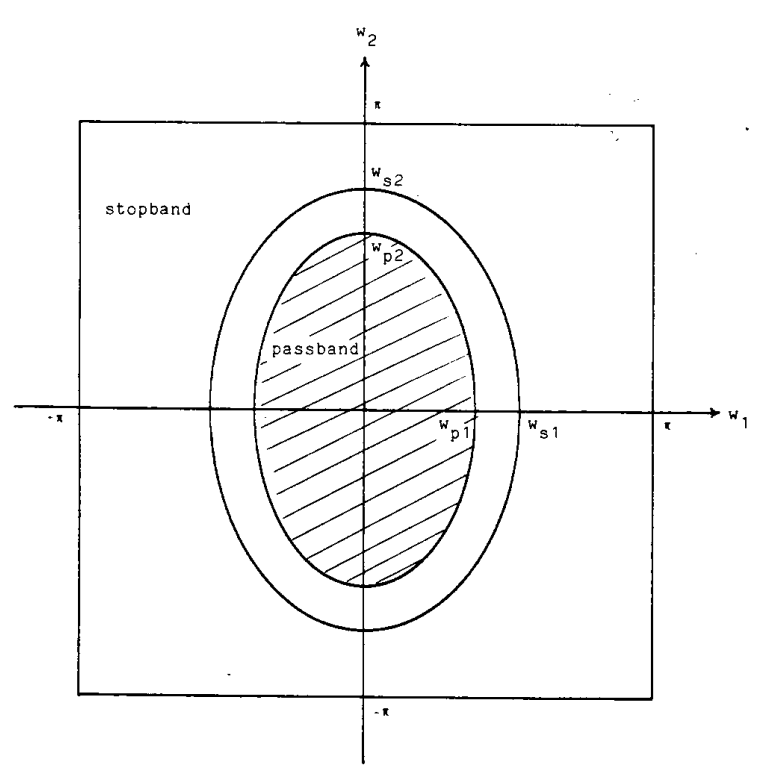

(a)

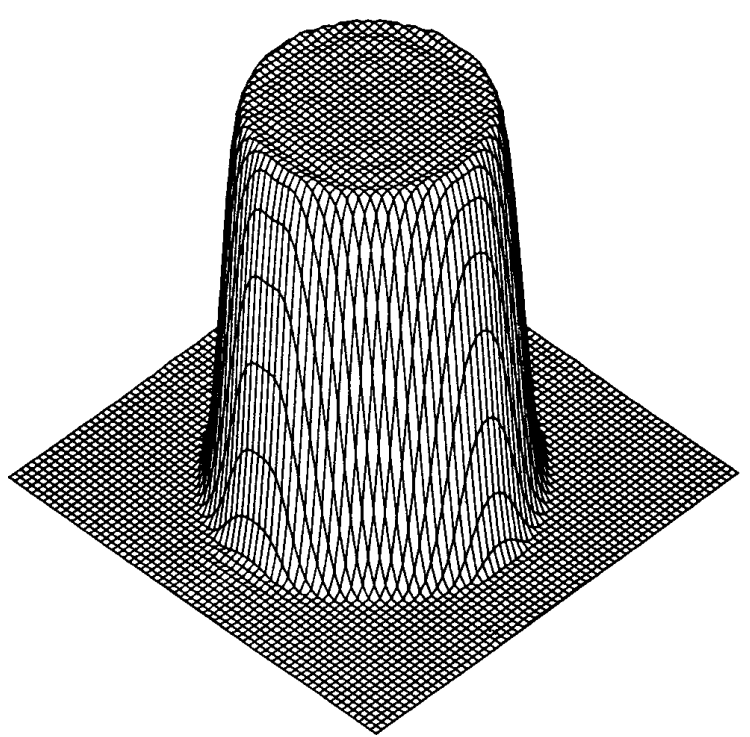

(b)

Fig. 2. (a) Specification of 2-D circular and elliptical low-pass filters in Example 2.2. (b) A $25 \times 25$ 2-D circular low-pass filter with $w_{p 1}=w_{p 2}$ $=0.5 \pi, w_{s 1}=w_{s 2}=0.7 \pi$, and $\alpha=\beta=1$ in Example 2.2.

in which $0_{2}$ denotes a zero matrix with dimension $N \times$ $(N+1) / 2$ and

$$
D_{1}=\left[\begin{array}{lllll}
0 & 0 & 0 & \cdots & 0.25 \\
\vdots & \vdots & \vdots & \ddots & \vdots \\
0 & 0 & 0.25 & \cdots & 0 \\
0 & 0.25 & 0 & \cdots & 0 \\
0.5 & 0 & 0 & \cdots & 0 \\
0 & 0.25 & 0 & \cdots & 0 \\
0 & 0 & 0.25 & \cdots & 0 \\
\vdots & \vdots & \vdots & \ddots & \vdots \\
0 & 0 & 0 & \cdots & 0.25
\end{array}\right] .
$$


TABLE I

2-D AND 3-D FIR FILTER DESIGN EXAMPLES

\begin{tabular}{|c|c|c|c|c|c|c|c|}
\hline Example & Type of $2 D$ filter & Length & $\begin{array}{l}\text { Method for } \\
\text { establishing } 0\end{array}$ & $\begin{array}{l}\text { Peak error } \\
\text { in passband }\end{array}$ & $\begin{array}{l}\text { Peak error } \\
\text { in stopband }\end{array}$ & $\begin{array}{l}\text { Design time } \\
\text { on } V A X-11 / 780\end{array}$ & $\begin{array}{l}\text { Illustrated } \\
\text { figure }\end{array}$ \\
\hline 2.1 & $\begin{array}{l}\text { rectangular } \\
\text { low-pass }\end{array}$ & $27 \times 27$ & closed form & $\begin{array}{l}0.005826 \\
(0.051 \mathrm{~dB})\end{array}$ & $\begin{array}{l}0.003607 \\
\left(-48.86^{\mathrm{d}} \mathrm{d}\right)\end{array}$ & $10.63 \mathrm{~min}$. & 1 \\
\hline 2.2 & $\begin{array}{l}\text { circular } \\
\text { low-pass }\end{array}$ & $25 \times 25$ & $\begin{array}{l}\text { numerical } \\
\text { integration }\end{array}$ & $\begin{array}{l}0.006804 \\
(0.059 \mathrm{~dB})\end{array}$ & $\begin{array}{l}0.007398 \\
(-42.62 \mathrm{~dB})\end{array}$ & $33.54 \mathrm{~min}$. & 2 \\
\hline $2 \cdot 3$ & fan filter & $23 \times 23$ & closed form & $\begin{array}{l}0.043028 \\
(0.366 \mathrm{~dB})\end{array}$ & $\begin{array}{l}0.21395 \\
(+33.39 \mathrm{~dB})\end{array}$ & $16.18 \mathrm{~min}$. & 3 \\
\hline 2.4 & $\begin{array}{l}\text { minimum-energy } \\
\text { stopband }\end{array}$ & $15 \times 15$ & $\begin{array}{l}\text { numerical } \\
\text { integration }\end{array}$ & don't care & $\begin{array}{l}0.002451 \\
(-52.21 \mathrm{~dB})\end{array}$ & $3.38 \mathrm{~min}$. & 4 \\
\hline 3.1 & $\begin{array}{l}\text { elliptical } \\
\text { low-pass }\end{array}$ & $19 \times 19$ & $\begin{array}{l}\text { numerical } \\
\text { integration }\end{array}$ & 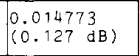 & $\begin{array}{l}0.009442 \\
(-40.50 \mathrm{~dB})\end{array}$ & $15.35 \mathrm{~min}$ & 5 \\
\hline 3.2 & $\begin{array}{l}2-D \text { symmetric } \\
\text { around }\left(w_{1}, w_{2}\right)=\left(\frac{\pi}{2}, \frac{\pi}{2}\right)\end{array}$ & $29 \times 29$ & closed form & $\begin{array}{l}0.009527 \\
(0.082 \mathrm{~dB})\end{array}$ & $\mid \begin{array}{l}0.009193 \\
(-40.73 \mathrm{~dB})\end{array}$ & $27.72 \mathrm{sec}$ & 6 \\
\hline 3.3 & $\begin{array}{l}\text { conic } \\
\text { low-pass }\end{array}$ & $27 \times 27$ & $\begin{array}{l}\text { numerical } \\
\text { integration }\end{array}$ & $\begin{array}{l}0.004165 \\
(0.036 \mathrm{~dB})\end{array}$ & $\left(\begin{array}{l}0.003039 \\
(-50.34 \mathrm{~dB})\end{array}\right.$ & $32.01 \mathrm{~min}$. & 7 \\
\hline 4.1 & $\begin{array}{l}\text { 2-D half-band } \\
\text { filter }\end{array}$ & $31 \times 39$ & closed form & $\begin{array}{l}0.003528 \\
(0.031 \mathrm{~dB})\end{array}$ & $\begin{array}{l}0.003528 \\
(-49.05 \mathrm{~dB})\end{array}$ & $22.15 \mathrm{sec}$. & 8 \\
\hline 5.1 & $\begin{array}{l}\text { circuiar } \\
\text { band-pass }\end{array}$ & $27 \times 27$ & $\begin{array}{l}\text { numerical } \\
\text { integration }\end{array}$ & $\begin{array}{l}0.004966 \\
(0.043 \mathrm{~dB})\end{array}$ & {$\left[\begin{array}{l}0.031156 \\
(-30.13 \mathrm{~dB})\end{array}\right.$} & $46.81 \mathrm{~min}$. & 10 \\
\hline 5.2 & $\begin{array}{l}\text { 3-D sphere } \\
\text { low-pass }\end{array}$ & $11 \times 11 \times 11$ & $\begin{array}{l}\text { numerical } \\
\text { integration }\end{array}$ & $\begin{array}{l}0.083847 \\
(0.699 \mathrm{~dB})\end{array}$ & $\begin{array}{l}0.075536 \\
(-22.44 \mathrm{~dB})\end{array}$ & $83.48 \mathrm{~min}$. & 11 \\
\hline
\end{tabular}

Notice that the dimension of $D$ is $N^{2} \times((N+1) / 2)^{2}$ and that of $D_{1}$ is $N \times(N+1) / 2$. Thus (17) becomes

$$
E_{N}=\frac{A^{t} D^{t} S^{t} S D A}{\hat{s}^{t} \cdot \hat{s}}=A^{t} \cdot Q_{N} \cdot A
$$

and the overall quadratic objective function to be minimized is therefore

$$
E=\alpha E_{p}+\beta E_{s}+\gamma E_{N}=A^{t}\left[\alpha Q_{p}+\beta Q_{s}+\gamma Q_{N}\right] A .
$$

The specification of such a 2-D elliptical filter can be referred to Fig. 2(a), if $N_{1}=N_{2}=19, w_{p 1}=0.3 \pi, w_{p 2}=$ $0.7 \pi, w_{s 1}=0.5 \pi, \quad w_{s} 2=0.9 \pi, \alpha=\beta=1, r=10^{-3}, L=11$, and the unwanted waveform $s(i, j)$ is $11 \times 11$ in length with the following type:

$$
s(i, j)=0.01 \sin \left(\sqrt{i^{2}+j^{2}} / 15\right), \quad 0 \leqslant i, j \leqslant 10
$$

using eigenfilter approach, the resultant $2-\mathrm{D}$ elliptical filter which rejects the above waveform is shown in Fig. 5.

\section{Example 3.2: Design of 2-D Filters with Some of Impulse Response are Constrained to Zero}

In 1-D filters, if the frequency response is symmetrical around $w=\pi / 2$, then the every other impulse response sample is exactly zero [6], we can extend this to the 2-D cases, for example, the 2-D frequency response shown in Fig. 6 is symmetric about both $W_{1}=\pi / 2$ and $W_{2}=\pi / 2$ in the first quadrant, hence the 2-D impulse response for $N$ odd

$$
h\left(n_{1}, n_{2}\right)=0, \quad \text { for } n_{1} \text { odd or } n_{2} \text { odd. }
$$

Thus we can delete the zero elements in column vector $A$ and eliminate their corresponding rows and columns in matrix $Q$, the order of matrix $Q$ is greatly reduced, and we can save a lot of computation time in this filter design.

\section{Example 3.3: Design of 2-D Circular Conic Filter}

For the above examples, all of the desired frequency responses in passband are unity again. In this example, a
2-D circular conic filter is designed with the desired amplitude response as

$$
D\left(w_{1}, w_{2}\right)= \begin{cases}1-\frac{\sqrt{w_{1}^{2}+w_{2}^{2}}}{w_{p}}, & w_{1}^{2}+w_{2}^{2} \leqslant w_{p}^{2} \\ 0, & \text { otherwise. }\end{cases}
$$

By substituting (26) into (10b) and setting $N_{1}=N_{2}=27$, $w_{p}=0.56 \pi, \alpha=\beta=1$, and $\left(w_{10}, w_{20}\right)=(0,0)$, we get the resultant amplitude response shown in Fig. 7 designed by eigenapproach.

\section{2-D Half-Band Filter Design}

Recently, an improved method to design 1-D half-band filters is developed by Vaidyanathan and Nguyen [7], by first designing a one-band prototype filter $G(Z)$ of order $(N-1) / 2$, whose passband extends from 0 to $2 w_{p}$ and the transition band is from $2 w_{p}$ to $\pi$ with no stopband, then the 1-D half-band filter $H(Z)$ is related to $G(Z)$ by

$$
H(Z)=\frac{G\left(Z^{2}\right)+Z^{-(N-1) / 2}}{2} .
$$

Then the stopband cutoff frequency $w_{s}=\pi-w_{p}$ and the passband and stopband deviations $\delta_{p}(w)$ and $\delta_{s}(w)$ satisfy $\delta_{p}(w)=\delta_{s}(\pi-w)$ automatically, this improved method greatly reduces the design time by a considerable amount [7].

Similarly for 2-D case, we can first design a one-band 2-D square filter $G\left(Z_{1}, Z_{2}\right)$ where the cutoff frequencies $w_{p 1}=w_{p 2}$ and the rest region is the transition band with no stopband, then construct a 2-D half-band filter by

$$
H\left(Z_{1}, Z_{2}\right)=\frac{G\left(Z_{1}^{2}, Z_{2}^{2}\right)+Z_{1}^{-(N-1) / 2} Z_{2}^{-(N-1) / 2}}{2}
$$

and the impulse response of $H\left(Z_{1}, Z_{2}\right)$ is related to that of 


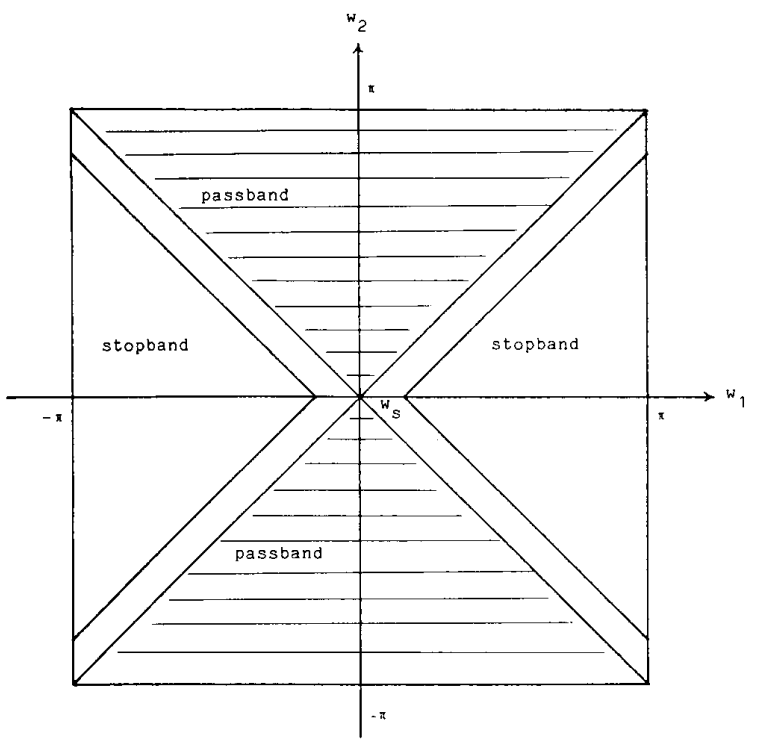

(a)

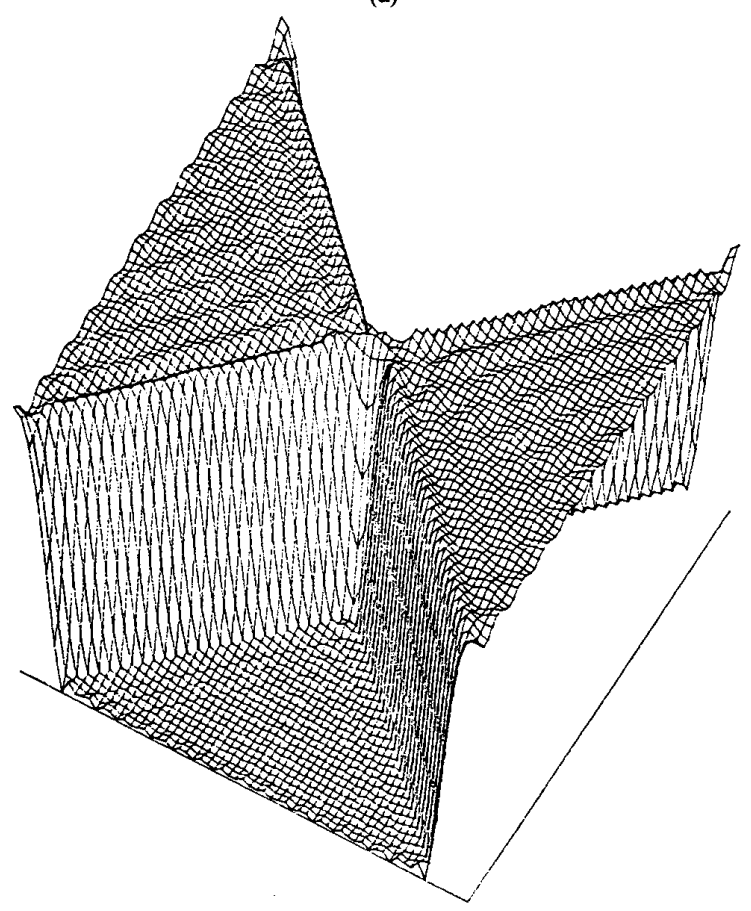

(b)

Fig. 3. (a) Specification of 2-D fan-type filter in Example 2.3. (b) A $23 \times 232$-D fan-type filter with $w_{s}=0.16 \pi, \alpha=\beta=1$, and $\left(w_{10}, w_{20}\right)=$ $(0, \pi / 2)$ in Example 2.3.

$$
\begin{aligned}
& G\left(Z_{1}, Z_{2}\right) \text { by } \\
& h\left(n_{1}, n_{2}\right)= \begin{cases}\frac{1}{2} g\left(\frac{n_{1}}{2}, \frac{n_{2}}{2}\right), & n_{1}, n_{2} \text { even } \\
\frac{1}{2}, & n_{1}=n_{2}=(N-1) / 2 \\
0, & \text { otherwise }\end{cases}
\end{aligned}
$$

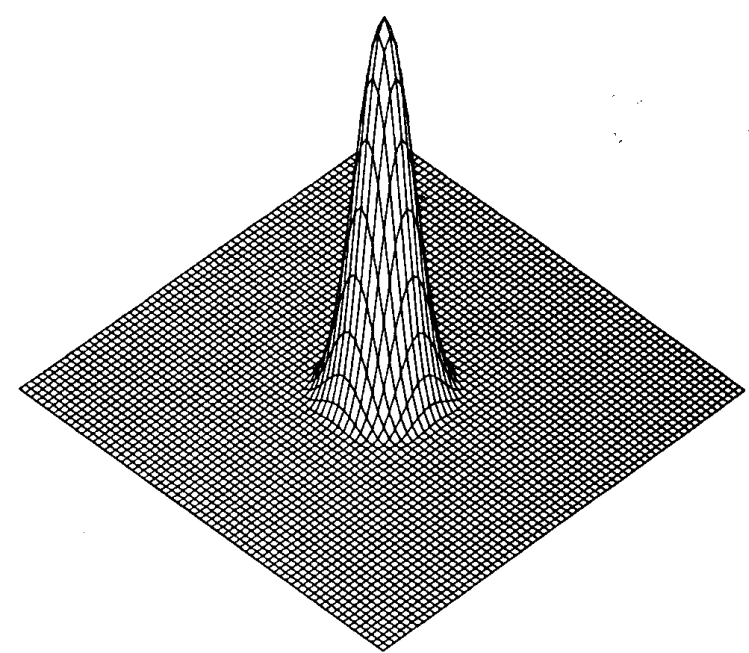

Fig. 4. A $15 \times 15$ 2-D filter with minimum energy stopband, in which the don't care band is a circular region with radius $0.3 \pi$, and $\alpha=0$, $\beta=1$ in Example 2.4

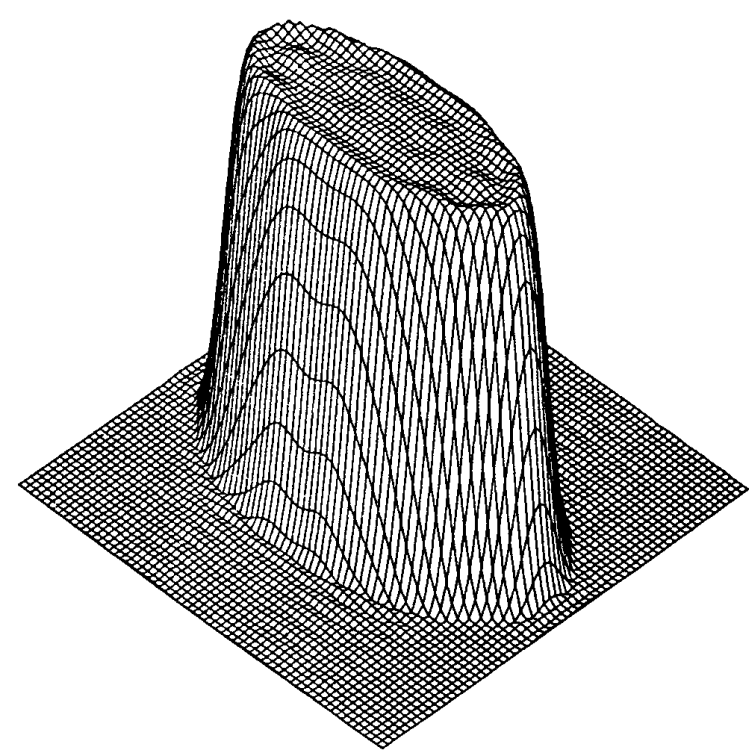

Fig. 5. A $19 \times 192$-D elliptical filter with $w_{p 1}=0.3 \pi, w_{p 2}=0.7 \pi, w_{s 1}=$ $0.5 \pi, w_{s 2}=0.9 \pi, \alpha=\beta=1, \gamma=10^{-3}$, which can reject $s(i, j)$ waveform of (24) in Example 3.1 .

and the 2-D passband and stopband deviations are

$$
\begin{aligned}
\delta_{p}\left(w_{1}, w_{2}\right) & =\delta_{p}\left(\pi-w_{1}, \pi-w_{2}\right) \\
& =\delta_{s}\left(w_{1}, \pi-w_{2}\right)=\delta_{s}\left(\pi-w_{1}, w_{2}\right)
\end{aligned}
$$

\section{Example 4.1: Design of 2-D Half-Band Filter}

Fig. 8(a) shows a one-band 2-D square filter $G\left(Z_{1}, Z_{2}\right)$ with length $16 \times 16$ and $w_{p 1}=w_{p 2}=0.84 \pi$, and the corresponding 2-D half-band filter is designed and shown in Fig. 8(b). 


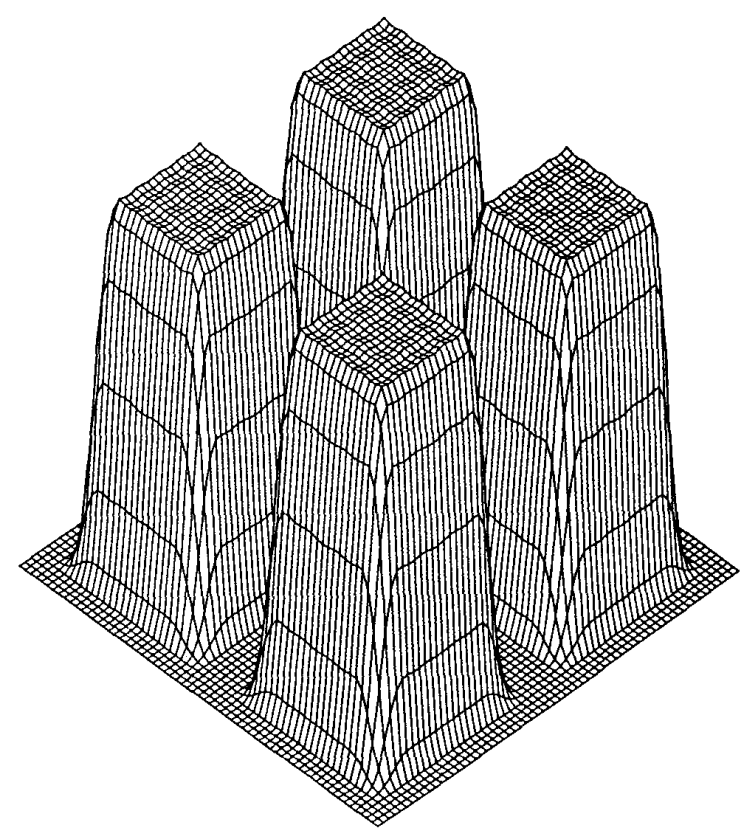

Fig. 6. A $29 \times 29$ 2-D filter whose frequency response is symmetric about $\left(w_{1}, w_{2}\right)=(\pi / 2, \pi / 2)$ in the first quadrant and $\alpha=\beta=1$ in

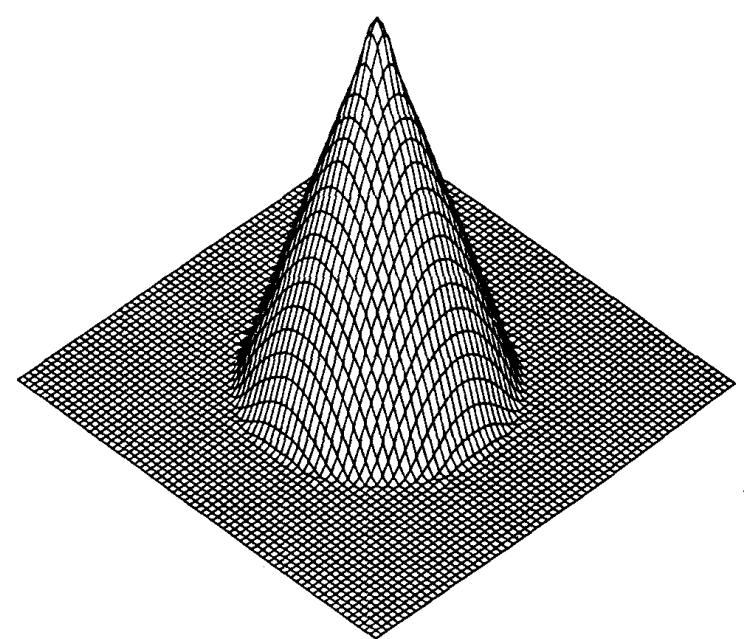

Fig. 7. A $27 \times 272$-D circular conic filter with $w_{p}=0.56 \pi$ and $\alpha=\beta=1$ in Example 3.3.

\section{COMPARISONS AND Discussions ON PERFormance}

To demonstrate the eigen-design approach, we make a detailed comparison of this method with the well-known McClellan transformation method [5] in terms of passband ripple, stopband attenuation, filter contour, and design time. The characteristics of all the examples are summarized in Table I; It is to be noted that design time on VAX $11 / 780$ computer is shorter if $q(n, m)$ integration can be formulated in a closed form, but it is generally longer if numerical integration is needed to use.
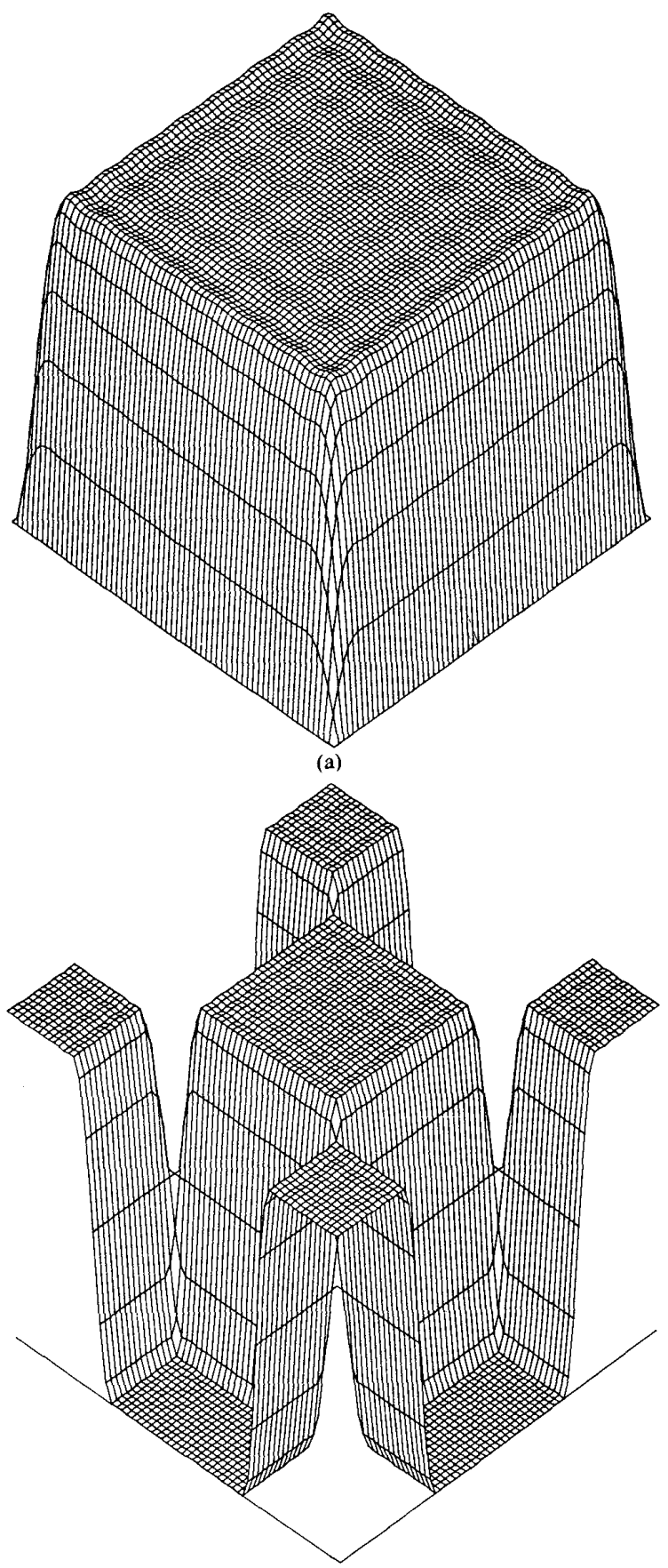

(b)

Fig 8. (a) A $16 \times 16$ one-band 2-D square filter with $w_{p 1}=w_{2}=0.84 \pi$ in Example 4.1. (b) A $31 \times 312-\mathrm{D}$ half-band filter corresponding to Fig. 8(a) in Example 4.1.

Fig. 9(a) and (b) presents the comparison of passband ripple between the eigen-approach (solid line) with the McClellan transformation method (dotted line). We use a 2-D circular low-pass filter with the same specification as in Example 2.2 for comparison, and the 1-D prototype filter for McClellan transformation is designed by the 

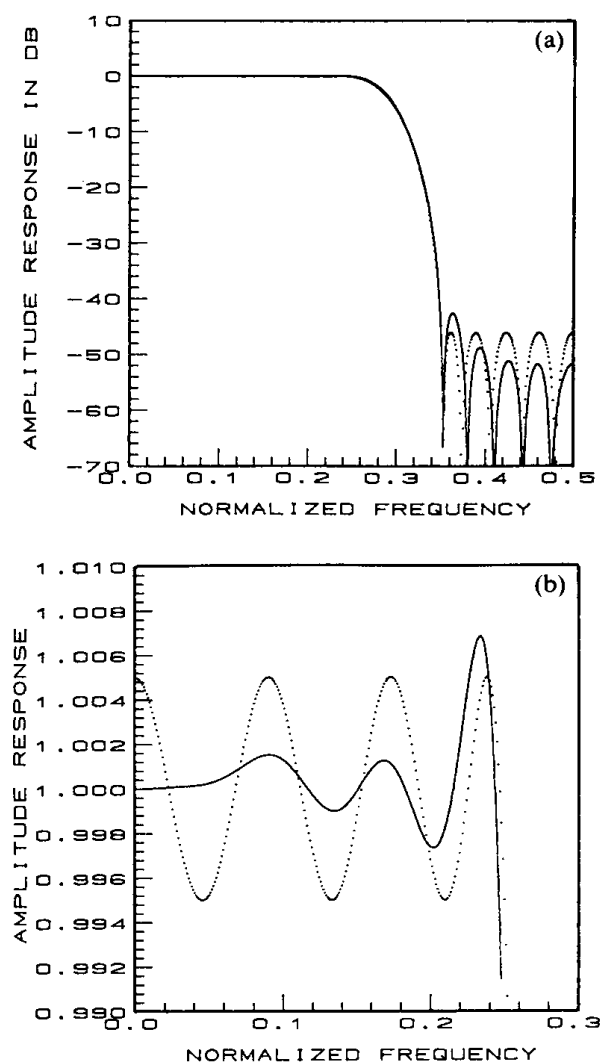

Fig. 9. (a) The frequency responses of the 2-D eigenfilter (solid line) and the McClellan transformed filter (dotted line) along the $w_{1}$-axis and $w_{2}=0$ in Section V. (b) The passband responses of Fig. 9 (a) in detail: eigenfilter (solid line), McClellan transformed filter (dotted line).

well-known Parks-McClellan program [2]. Fig. 9(a) shows both frequency responses of two filters along the $w_{1}$-axis and $w_{2}=0$, while Fig. 9 (b) gives the passband responses in detail. The eigenfilter approach is optimal in the least square sense. Comparison with the McClellan-Parks algorithm [2] for minimax equiripple filters shows that both are optimal in the sense of different minimum norms of the error function. However, a better performance is obtained with eigenapproach in most of the frequency band but not in the narrow-band region near the cutoff edge [8], [9]. It is interesting to study the choice of the reference frequency. We have made extensive computer simulations [8] and have avoided choosing the reference frequency at the band edge in the design. However, a better choice is to choose the reference frequency at the center of the band [8].

\section{Example 5.1: 2-D Circular Bandpass Filter Design and Contour Comparison}

About the 2-D filter's contour comparison, we take a 2-D circular bandpass filter with $N_{1}=N_{2}=27, w_{p 1}=$ $0.4 \pi, w_{p 2}=0.6 \pi, w_{s 1}=0.2 \pi, w_{s 2}=0.8 \pi$ as an example. Fig. 10(a) shows the resultant 2-D filter designed by eigenfilter approach and its corresponding contour is given in Fig.

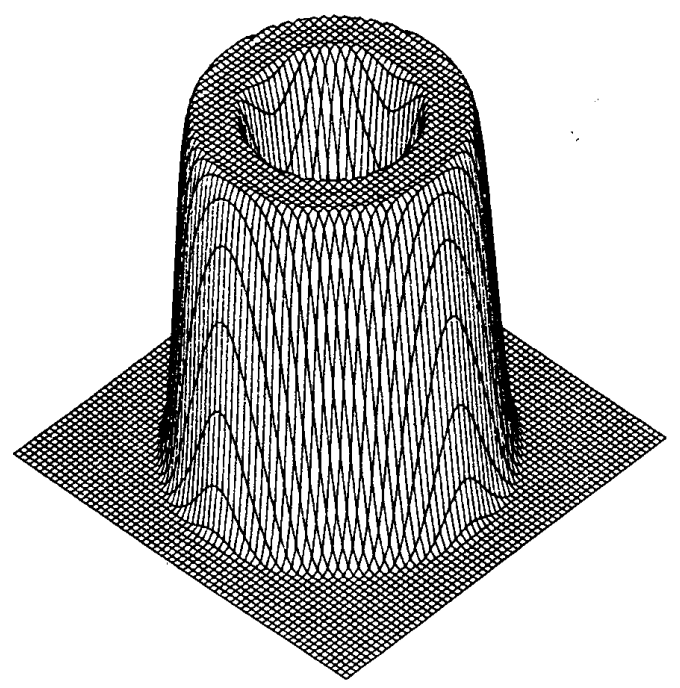

(a)

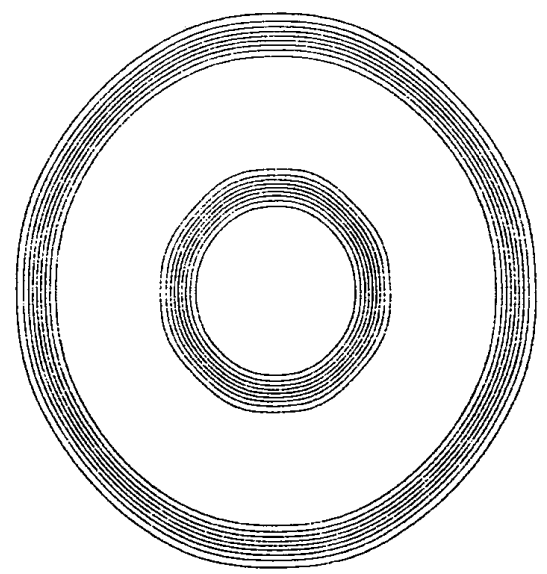

(b)

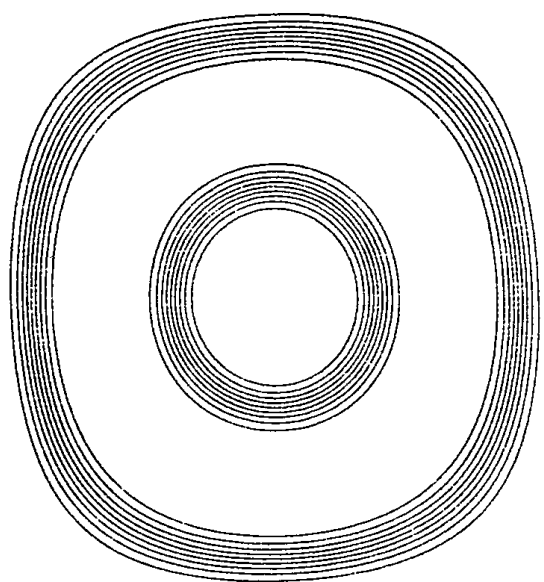

(c)

Fig. 10. (a) A $27 \times 27$ circular bandpass eigenfilter with $w_{p 1}=0.4 \pi$, $w_{p 2}=0.6 \pi, w_{s 1}=0.2 \pi, w_{s 2}=0.8 \pi$ for contour comparison in Example
5.1 . (b) The circular contours of 2-D eigenfilter in Fig. 10(a). (c) The circular contours of 2-D McClellan transformed filter with distortions in high frequency region. 
10(b), while Fig. 10(c) presents the contour of that designed by McClellan transformation. Notice that the contours in Fig. 10(c) are no longer circles and become square shape for higher frequencies and the eigenfilter contours in Fig. 10(b) are not distorted.

\section{Example 5.2: 3-D Sphere Low-Pass Filter Design}

The eigenfilter approach can be fairly easily extended to higher dimension digital filters, as an example, the frequency response of a 3-D $N \times N \times N$ octogonal symmetric filter for $N$ odd can be formulated as follows:

$$
\begin{aligned}
& H\left(e^{j w_{1}}, e^{j w_{2}}, e^{j w_{3}}\right)=\exp \left(-j \frac{N-1}{2}\right)\left(w_{1}+w_{2}+w_{3}\right) \\
& \text { - } \left.\sum_{n_{1}=0}^{\frac{N-1}{2}} \sum_{n_{2}=0}^{\frac{N-1}{2}} \sum_{n_{3}=0}^{\frac{N-1}{2}}\right) a\left(n_{1}, n_{2}, n_{3}\right) \\
& \cdot \cos n_{1} w_{1} \cdot \cos n_{2} w_{2} \cos n_{3} w_{3}
\end{aligned}
$$

where $a\left(n_{1}, n_{2}, n_{3}\right)$ is related to the impulse response $h\left(n_{1}, n_{2}, n_{3}\right)$ by

$$
\begin{aligned}
& a(0,0,0)=h\left(\frac{N-1}{2}, \frac{N-1}{2}, \frac{N-1}{2}\right) \\
& a\left(n_{1}, 0,0\right)=2 h\left(\frac{N-1}{2}-n_{1}, \frac{N-1}{2}, \frac{N-1}{2}\right), \\
& a\left(0, n_{2}, 0\right)=2 h\left(\frac{N-1}{2}, \frac{N-1}{2}-n_{2}, \frac{N-1}{2}\right), \cdots, \frac{N-1}{2} \\
& a\left(0,0, n_{3}\right)=2 h\left(\frac{N-1}{2}, \frac{N-1}{2}, \frac{N-1}{2}-n_{3}\right), \\
& a\left(n_{1}, n_{2}, 0\right)=4 h\left(\frac{N-1}{2}-n_{1}, \frac{N-1}{2}-n_{2}, \frac{N-1}{2}\right), \\
& a\left(0, n_{2}, n_{3}\right)=4 h\left(\frac{N-1}{2}, \frac{N-1}{2}-n_{2}, \frac{N-1}{2}-n_{3}\right) \\
& a\left(n_{1}, 0, n_{3}\right)=4 h\left(\frac{N-1}{2}-n_{1}, \frac{N-1}{2}, \frac{N-1}{2}-n_{3}\right) \\
& n_{1}, n_{2}=1, \cdots, \frac{N-1}{2} \\
& n_{1}, n_{3}=1, \cdots, \frac{N-1}{2}
\end{aligned}
$$

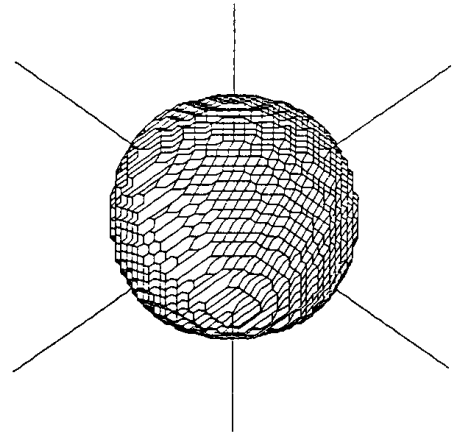

(a)

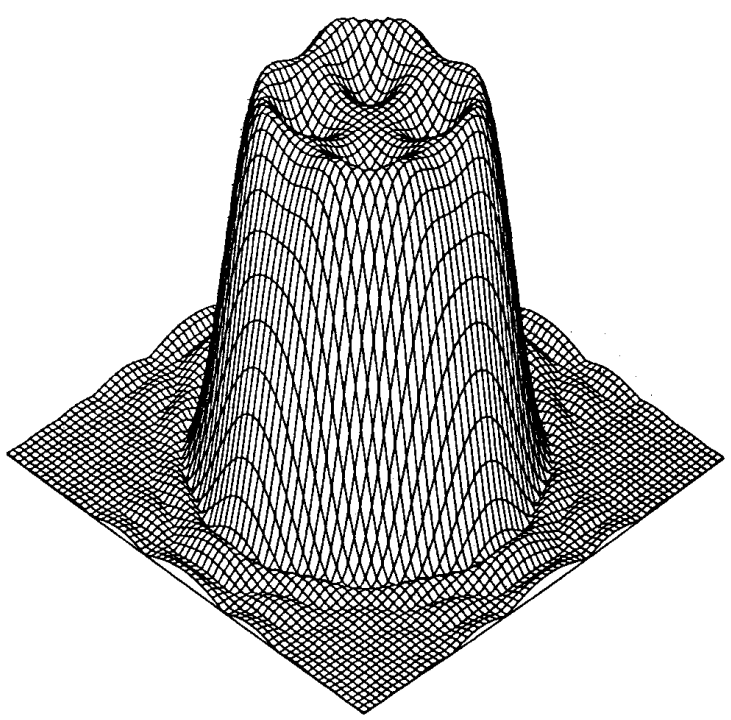

(b)

Fig. 11. (a) The 3 - $d B$ gain surface of a $11 \times 11 \times 113$ - $D$ sphere low-pass filter with $w_{p}=0.5 \pi, w_{s}=0.7 \pi, \alpha=\beta=1$ in Example 5.2. (b) The frequency response of the 3-D low-pass filter in Fig. 11(a) along the $w_{1}, w_{2}$-axis plane and $w_{3}=0$

By modifying the technique stated in Sections II and III, the error function can be formulated in the same way to design a 3-D filter. Fig. 11(a) shows the 3-dB gain surface of a $11 \times 11 \times 113$-D sphere low-pass filter with $w_{p}=$ $0.5 \pi, w_{s}=0.7 \pi, \alpha=\beta=1$, and the reference frequency point $\left(w_{10}, w_{20}, w_{30}\right)=(0,0 ., 0)$, Fig. 11(b) shows the frequency response of the 3-D filter along the $w_{1}, w_{2}$-axis plane, and $w_{3}=0$.

\section{Conclusions}

In this paper, the 1-D eigenfilter approach is extended for designing 2-D FIR filters; This approach is simple and powerful, and can be fairly easily extended to higher dimension digital filters. Several numerical design exam- 
ples are used to illustrate the effectiveness of this approach.

\section{REFERENCES}

[1] P. P. Vaidyanathan and T. Q. Nguyen, "Eigenfilters: A new approach to least-squares FIR filter design and applications including Nyquist filters," IEEE. Trans. Circuits Syst., vol. CAS-34, pp. 11-23, Jan. 1987.

[2] J. H. McClellan, T. W. Parks, and L. R. Rabiner, "A computer program for designing optimum FIR linear phase digital filters," program for designing optimum FIR linear phase digital filters,"
IEEE Trans. A udio. Electroacoust., vol. AU-21, pp. 506-526, Dec. 1973.

[3] A. Nashashibi and C. Charalambous, "2-D FIR eigenfilters," in Proc. IEEE Int. Symp. on Circuits and Systems, pp. 1037-1040, Helsinki, Finland, June 1988

[4] B. Nobel and J. W. Daniel, Applied Linear Algebra, Englewood Cliffs, NJ: Prentice-Hall, 1977.

[5] J. H. McClellan, "The design of two-dimensional digital filters by transformations," in Proc. 7 th Princeton Conf. on Inform. Sci. and Syst., pp. 247-251, 1973

[6] L. R. Rabiner and B. Gold, Theory and Application of Digital Signal Processing. Englewoods Cliffs, NJ: Prentice-Hall pp. 472-478, pp. $170-171,1975$.

[7] P. P. Vaidyanathan and T. Q. Nguyen, "A trick for the design of FIR half-band filters," IEEE Trans. Circuits Syst, vol. CAS-34, pp. 297-300, Mar. 1987

[8] S. C. Pei and J. J. Shyu, "Eigenfilter design of higher-order digital differentiators," IEEE Trans. Acoust. Speech, Signal Processing, vol. 37, pp. 505-511, Apr. 1989.

[9] "Design of FIR Hilbert transformers and differentiators by eigenfilter," IEEE Trans. Circuits Syst., vol. 35, pp. 1457-1461, Nov. 1988.

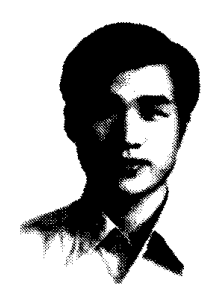

Jong-Jy Shyu ( $\mathrm{S}^{\prime} 88$ ) received the B.S. degree from Tatung Institute Technology, Taipei, in 1983, and the M.S. degree from National Taiwan University, Taipei, in 1988, both in electrical engineering. $\mathrm{He}$ is currently working towards the $\mathrm{Ph} . \mathrm{D}$. degree in electrical engineering at $\mathrm{Na}$ tional Taiwan University.

His research interests include filter design, digital signal processing, and image processing.

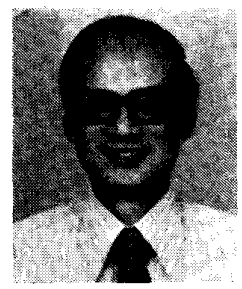

Soo-Chang Pei (M'86) received the B.S. degree from National Taiwan University in 1970 and the M.S. and Ph.D. degree from the University of California, Santa Barbara, in 1972 and 1975 , respectively, all in electrical engineering.

He was an engineering officer in the Chinese Navy Shipyard at Peng Fu Island from 1970 to 1971 and a Research Assistant at the University of California, Santa Barbara from 1971 to 1975. $\mathrm{He}$ was Professor and Chairman in the Department of Electrical Engineering at Tatung Institute of Technology from 1981 to 1983 . At present he is a Professor with the Department of Electrical Engineering at National Taiwan University. His research interests include digital signal processing, digital picture processing, optical information processing, laser, and holography.

Dr. Pei is a member of Eta Kappa Nu and the Optical Society of America. 